\title{
A novel technique for modified all-inside repair of bucket-handle meniscus tears using standard arthroscopic portals
}

\author{
Jing Hui Yik, Bryan Thean Howe Koh ${ }^{*}$ and Wilson Wang
}

\begin{abstract}
Background: Bucket-handle meniscus tears (BHMT) are often displaced and unstable. The inside-out technique of repairing such tears is currently the gold standard. All-inside repair with meniscal fixators is getting increasingly popular. Shortcomings of the inside-out technique include neurovascular complications, especially saphenous nerve palsy, and retention of a non-resorbable suture which can result in discomfort to patient, granuloma formation, and a foci of infection. Hence, the purpose of this project was to innovate a novel all-inside technique to precisely reduce and fix BHMT while avoiding neurovascular complications and retention of a non-resorbable suture.

Methods: Routine arthroscopic portals were created on a patient's left knee with a displaced BHMT. Through the anteromedial portal, a conjoint pseudo double lumen cannula was inserted. Two limbs of a reduction suture were passed through the cannula, one over the "femoral" surface of the meniscus, one over the "tibial" surface of the meniscus anterior to the biceps femoris tendon, with the knee flexed at $20^{\circ}$ to avoid injury to the saphenous nerve. Suture limbs were passed out percutaneously and tensioned.
\end{abstract}

Results: Anatomic reduction was ensured under arthroscopic visualization with ease. All inside repair was performed using the vertical mattress suture configuration. Reduction sutures were subsequently removed by cutting flush to the skin and pulling on one suture limb. The patient was back to full activities with minimal discomfort 8 months postoperatively.

Conclusion: The technique described is superior to existing techniques for the following reasons: (1) Reduction of the displaced meniscal tear is "extra-meniscal," avoiding further trauma to a damaged meniscus. (2) Tensioning of the two suture limbs created promotes better control of reduction through tensioning. (3) Risk of discomfort, infection, and neurovascular damage caused by a retained suture is reduced. (4) No additional portals/equipment is required. We encourage this novel technique to be attempted by surgeons.

Keywords: Arthroscopy, Bucket-handle meniscus tear, Novel technique

\section{Background}

The meniscus deepens the tibial articular surface, stabilizes the knee joint, allows load transmission, reduces articular contact stress, and aids in lubrication [11]. Meniscal repairs are preferable over partial or total meniscectomies as they aim to restore a functional meniscus and possibly prevent early degenerative changes $[7,13]$.

\footnotetext{
* Correspondence: bryankohth@hotmail.com

Department of Orthopaedic Surgery, National University Health Systems (NUHS), 1 E Kent Ridge Road, NUHS Tower Block Level 11, Singapore 119228, Singapore
}

(c) The Author(s). 2017 Open Access This article is distributed under the terms of the Creative Commons Attribution 4.0 International License (http://creativecommons.org/licenses/by/4.0/, which permits unrestricted use, distribution, and reproduction in any medium, provided you give appropriate credit to the original author(s) and the source, provide a link to the Creative Commons license, and indicate if changes were made. The Creative Commons Public Domain Dedication waiver (http://creativecommons.org/publicdomain/zero/1.0/) applies to the data made available in this article, unless otherwise stated. repairing bucket-handle meniscus tears (BHMT): allinside, inside-out, outside-in, and modifications of these techniques. The inside-out technique is the goldstandard and especially useful for middle third tears, posterior horn tears, and displaced BHMT. An incision is made either posteromedially or posterolaterally to access the capsule and allow sutures to be passed insideout under arthroscopic visualization. In the setting of an unstable, displaced BHMT, inside-out repair provides accurate reduction and strong fixation of the torn meniscus [1]. The outside-in technique is most useful for 
anterior horn tears but can also be employed for middle third tears and BHMT. All-inside repair with meniscal fixators is increasingly popular due to ease of use, reduced operative time, and reduction in neurovascular complications [4].

However, there are limitations and drawbacks to each technique. For the purposes of this article, we will focus on BHMT, which are typically displaced and unstable. The all-inside repair with meniscal fixators may not allow satisfactory coaptation and stable fixation for a BHMT.

Hybrid repairs with initial inside-out repair to reduce and provisionally fix the bucket handle fragment before subsequent all-inside repair with meniscal fixators can overcome this problem. The inside-out repair has several disadvantages: Firstly, it necessitates an incision and dissection down to capsule, with attendant risks of infections and neurovascular complications, as high as $21 \%$ [8]. Saphenous nerve palsy is the most neurologic complication encountered. Secondly, traditional insideout repair leaves a knot of non-resorbable suture material outside the capsule. This potentially leads to prominence of the knot, granuloma formation, and infection. Patients often complain of knot prominence as incisions are on the sides of the knee where extracapsular tissue layers are thin. However, this may be under-reported as it may not be considered a complication per se.

The authors introduce a modification of the hybrid repair for BHMT, using an inside-out reduction suture followed by all-inside repair with meniscal fixators. This avoids the disadvantages while retaining the advantages of conventional inside-out repair.

\section{Case summary}

A 34-year-old Chinese female with no previous notable medical or surgical history presented to clinic after sustaining an atraumatic twisting injury to the left knee while hiking. Post injury, she had pain on weight bearing, was unable to fully extend her left knee, and noted mild swelling. On physical examination, range of motion (ROM) was $0-140^{\circ}$, there was medial joint line tenderness, and the anterior drawer test was positive. MRI of the left knee showed a displaced bucket-handle medial meniscus tear in addition to a partial-thickness midsubstance ACL tear (Fig. 1).

The patient was subsequently counseled and consented for arthroscopic debridement and repair of the bucket-handle medial meniscus tear.

\section{Surgical technique}

\section{Diagnostic arthroscopic examination}

First, routine diagnostic arthroscopic examination of the knee joint was performed through standard anterolateral and anteromedial portals. In the setting of a displaced BHMT, provisional reduction was achieved through manipulation of the knee externally and manipulation of the torn meniscus fragment with an instrument such as an arthroscopic probe. The meniscus tear was assessed for size, location, chronicity, and pattern. Arthroscopic examination of the left knee confirmed a displaced bucket-handle medial meniscus tear involving the entire middle third and part of the posterior horn. The instruments used for the inside-out component was from the meniscal stitcher set for inside-out repair (Smith \& Nephew), while that used for the all-inside component was from the FAST-FIX 360 Meniscal Repair System (Smith \& Nephew) (Fig. 2).

\section{Passage of inside-out reduction sutures}

The next step involves passage of the inside-out reduction sutures above the femoral surface and below the tibial surface of the meniscus. The arthroscope was placed in the anteromedial portal, and a zone-specific
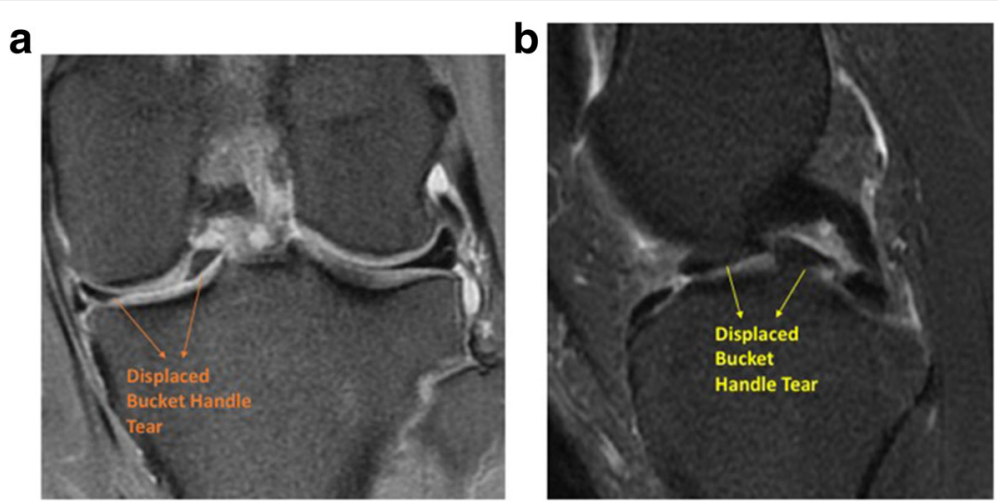

Fig. 1 Magnetic resonance imaging of the visualized meniscus tear. a Coronal proton density fat suppression image of the patient's left knee showing a displaced bucket-handle tear of the medial meniscus. $\mathbf{b}$ Sagittal T2 fat suppression image of the patient's left knee again showing the displaced medial meniscus bucket-handle tear 

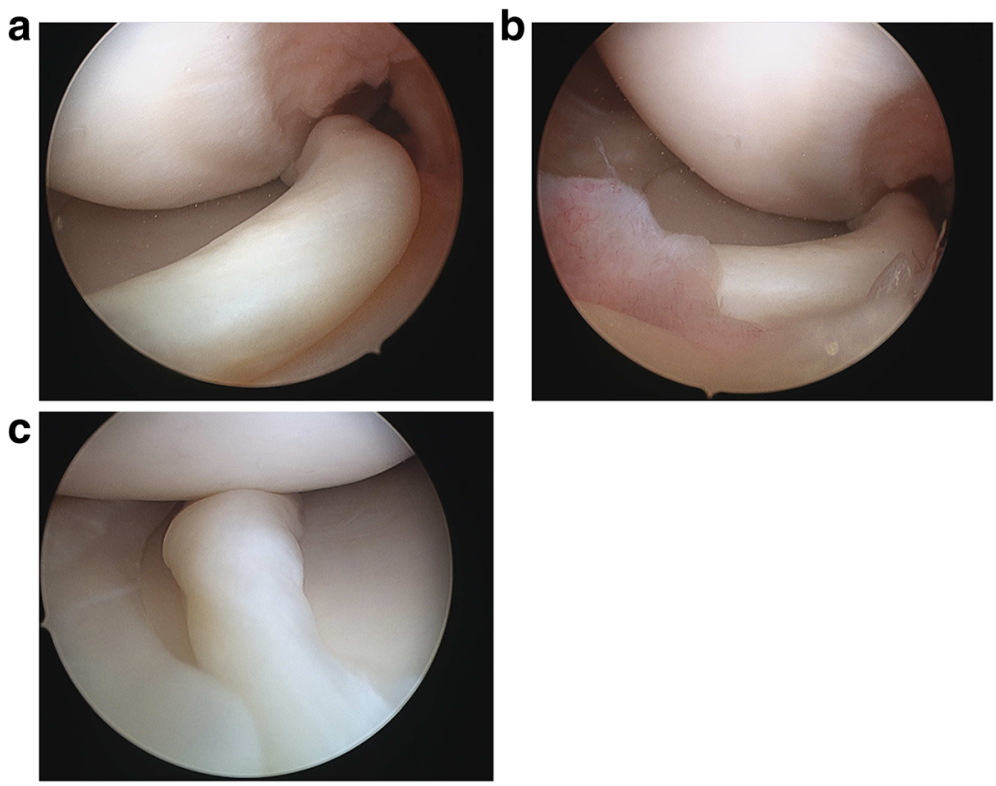

Fig. 2 Intra-arthroscopic visualization of the bucket-handle meniscus tear. a and $\mathbf{b}$ show a displaced bucket-handle meniscus tear (BHMT) in this patient extending from the middle to posterior third of the meniscus. $\mathbf{c}$ shows provisional reduction of the BHMT through blunt probe manipulation

double lumen cannula is inserted through the anterolateral portal. The cannula was positioned, and a flexible needle is passed through the first lumen of the cannula above the femoral surface of the meniscus. The needle then pierced out of the joint capsule with the knee in $20^{\circ}$ of flexion. The needle has an "open end" that allows a suture to be hooked on. After passage of the "femoral" limb of the suture, the other limb of the same suture was then hooked on to the needle. This needle was then passed through the second lumen of the cannula, below the tibial surface of the meniscus and out of the joint capsule with the knee in $20^{\circ}$ of flexion. This became the "tibial" limb of the suture. The double lumen cannula could now be withdrawn completely. The cannula used had a "conjoined" double lumen, which allowed the sequential passage of needles attached to both ends of a single suture, without subsequent obstruction to subsequent cannula withdrawal (Fig. 3).

The passage of the inside-out needles and sutures can be performed safely in a percutaneous manner for both medial and lateral meniscus tears. The critical structure to avoid on the medial side is the saphenous nerve, which emerges from the adductor canal to pierce the fascia lata between tendons of sartorius and gracilis before passing downwards on the medial side of the leg. With the knee in flexion, the saphenous nerve crosses the joint line at, or slightly behind the posteromedial corner of knee. The authors recommend passing the needle with the knee in $20^{\circ}$ of flexion and to keep the needle anterior to the posteromedial corner of the knee.
The critical structure to avoid on the lateral side is the common peroneal nerve, which is posterior to the biceps femoris tendon. The authors recommend passing the needle with the knee in $90^{\circ}$ of flexion and to keep the needle anterior to the biceps femoris tendon, which can be easily palpated. For effective stabilization of the tear, the reduction suture should be placed at the midpoint of the BHMT, which should be anterior to either the posteromedial corner of the knee or the biceps femoris tendon, thus allowing safe passage of the inside-out needles and sutures in a percutaneous manner.

\section{Achieving desired reduction and stabilization of the torn meniscus}

The two limbs of the suture were then tensioned simultaneously to achieve satisfactory reduction of the torn meniscus under arthroscopic visualization. A hemostatic artery was applied on the suture limbs, flush to skin, to maintain tension and, hence, reduction of the torn meniscus. A second inside-out reduction suture can also be used to attain anatomic reduction if required, bearing in mind the safe zones for passage of the inside-out needles (Fig. 4).

\section{All-inside repair with meniscal fixators}

Once satisfactory reduction and stabilization of the torn meniscus has been achieved with the reduction suture in place, all-inside repair with meniscal fixators can be performed. Multiple fixators are likely to be required for a BHMT. The authors recommend repairs starting with fixators immediately anterior and posterior to the 

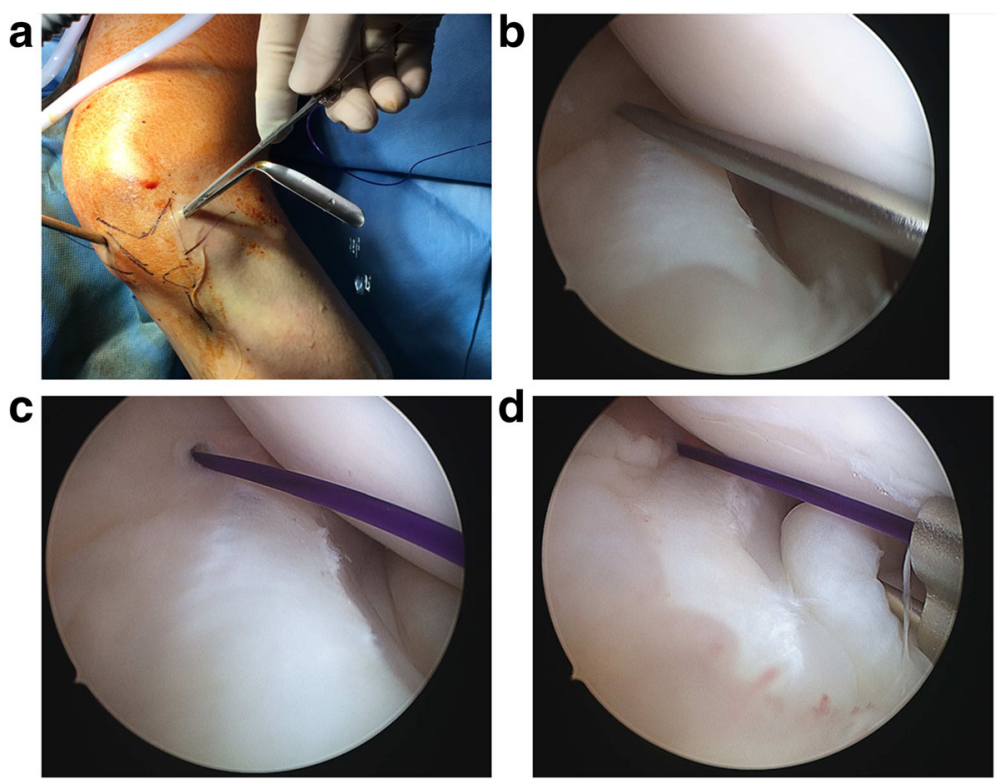

Fig. 3 Arthroscopic images showing initial reduction of the displaced meniscus. a shows positioning of the double lumen cannula. $\mathbf{b}$ and $\mathbf{c}$ show passage of the needle above the femoral surface of the meniscus, with care not to traumatize the meniscal substance. $\mathbf{d}$ shows passage of the second need below the tibial surface of the meniscus

reduction suture. Each subsequent fixator should then be placed progressively further away. It is also advised to place fixators on both the femoral and tibial sides of the tear to reduce puckering of the meniscus. The all-inside repairs can be performed in either vertical mattress or horizontal mattress suture configurations, although the vertical mattress is still considered the gold standard [10]. Finally, once meniscal repair is completed, the reduction suture can be removed by cutting either suture limb flush to the skin and pulling on the other suture limb. The reduction technique that we propose is illustrated in Figs. 5 and 6.
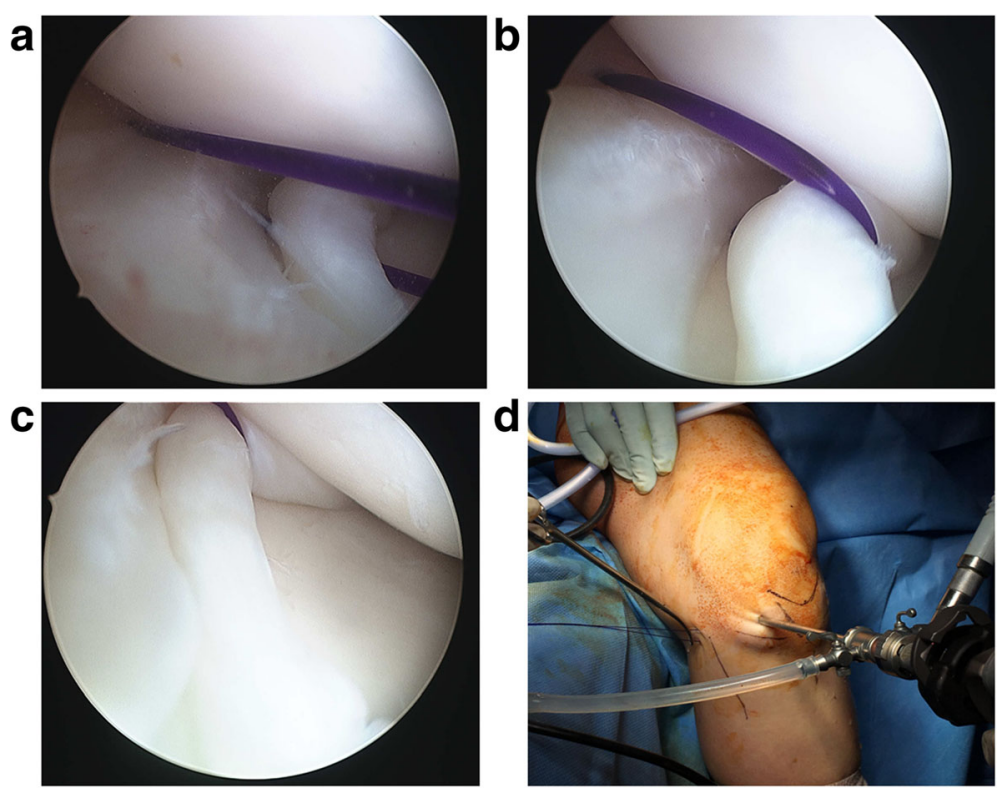

Fig. 4 Arthroscopic images showing tensioning of the reduced meniscus. a shows the superior and inferior inside-out reduction sutures in position with good placement. $\mathbf{b}$ and $\mathbf{c}$ show tensioning of the reduction sutures to achieve optimal reduction. $\mathbf{d}$ shows the use of artery forceps to maintain desired tension on the two reduction suture limbs 

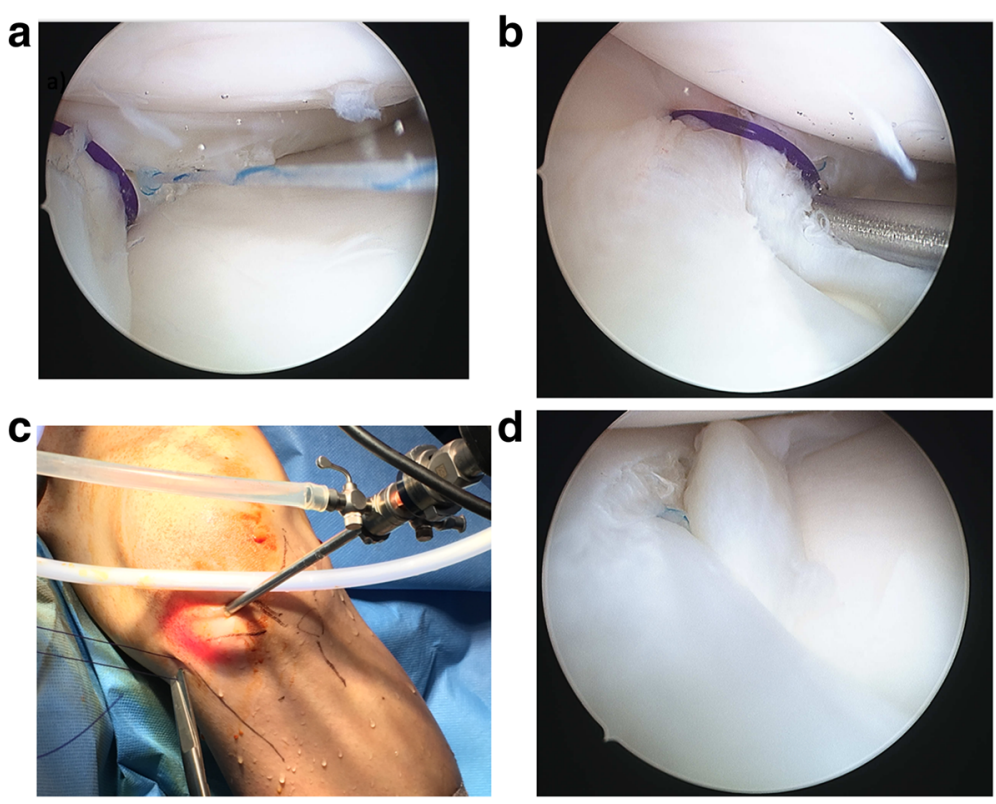

Fig. 5 Intra-operative images showing the final repair. a shows passing of the all-inside meniscus fixator posterior to the reduction suture. $\mathbf{b}$ shows passing of a second all-inside meniscus fixator anterior to the reduction suture. c shows cutting of one limb of the reduction suture flush to skin followed by removal of the reduction suture by pulling on the other side. $\mathbf{d}$ shows a well-positioned reduction of the BHMT with two all-inside meniscus fixators after removal of the reduction sutures

Six months post-operatively, the patient was pain free. Range of motion of her left knee was $0-130^{\circ}$. The anterior drawer test demonstrated a good firm endpoint. There was mild medial joint line discomfort but no tenderness on palpation. She was back to full sporting activities after 8 months.

\section{Discussion}

Meniscal tears are common injuries treated by orthopedic surgeons. Long-term follow-up studies have demonstrated increased arthritic changes after partial meniscectomy when compared with the anatomically normal contralateral knee [7]. The load transmitted across a knee joint increases with the amount of meniscus removed. As such, meniscal repairs are attempted in suitable patients (young; active) with suitable tears (simple longitudinal tears especially in the red-red/red-white zone) to try and restore the natural function and avoid early arthritic changes [13]. BHMT are often displaced and unstable, requiring inside-out suture repair with or without all-inside repair. The rationale for inside-out suture repair is that, unlike all-inside meniscal repair, it permits accurate reduction, stabilization, and coaptation of the tear edges [1]. The disadvantages of using an a

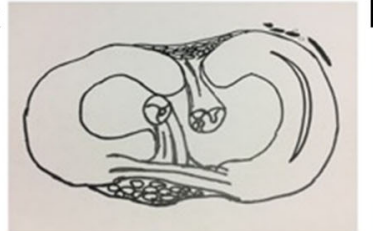

d

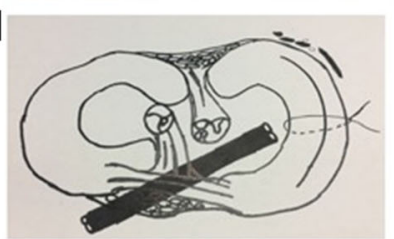

b

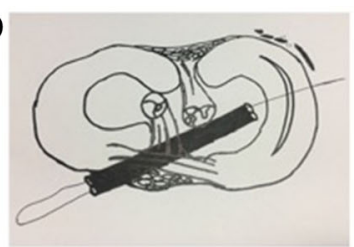

C

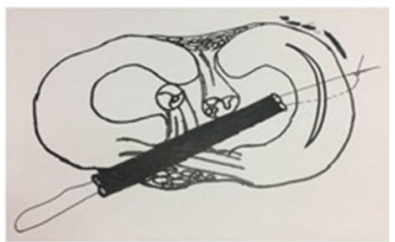

e

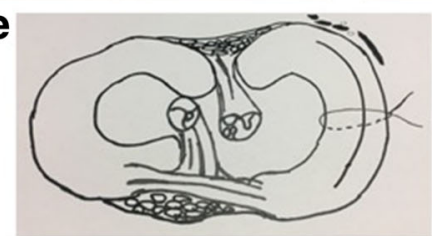

Fig. 6 Summary of illustrations of surgical technique. a Illustration showing medial meniscus buckle-handle meniscus tear. $\mathbf{b}$ Passing of the superior inside-out reduction suture. $\mathbf{c}$ Passing of the inferior inside-out reduction suture and tensioning of both reduction sutures. $\mathbf{d}$ and $\mathbf{e}$ Tensioning of both reduction sutures to achieve the desired reduction 
inside-out suture repair include the need for skin incisions either posterolaterally or posteromedially where there is risk of common peroneal nerve and saphenous nerve palsy, respectively. Overall risk of infections or neurovascular complications have been reported to be as high as $21 \%$ [8].

The proposed technique has several advantages over conventional inside-out repair. One feature of the proposed technique is that the inside-out reduction suture is used purely to achieve and maintain anatomic reduction of the torn meniscus while all-inside meniscus repair is done. It can be removed once meniscal repair is completed. This avoids the need for additional skin incisions because the suture does not need to be tied down onto the joint capsule, and hence, the risks of infection and neurovascular complications can be avoided. Avoiding the need for skin incisions and dissection down to capsule also potentially reduces operative time, [4] postoperative pain, and morbidity. Removing the suture after meniscus repair is completed also avoids any problems with knot prominence, granuloma, and infection that may be encountered with conventional inside-out repairs. Avoiding the need to tie sutures over the posterior capsule in this technique also avoids potential flexion contractures seen in conventional inside-out repair, which was reported by Morgan in 1991 [12].

Another feature of this technique is that the inside-out reduction suture is passed above the femoral surface and below the tibial surface of the meniscus. The position of the suture limbs above and below the meniscus, similar to a vertically oriented suture, also allows rotatory control. Tensioning of individual suture limbs may thus enable a more anatomic reduction. The conventional inside-out technique usually uses horizontal mattress sutures because vertical sutures are difficult to place; hence, it loses the rotatory control that is especially important in the case of an unstable, displaced BHMT where the torn fragment is often flipped or rotated. The technique proposed here allows rotatory control of the torn fragment through placement of the suture limbs above and below the meniscus in a vertical orientation. Ahn et al. [1] described a modified inside-out repair technique that involves passage of suture through the torn fragment to achieve rotatory control and improve reduction, but this may further damage the torn meniscus. The technique proposed here involves passing the inside-out reduction suture above and below the meniscus in an "extra-meniscal" manner, avoiding further trauma to a meniscus that is already damaged.

Advantages of all-inside repair techniques include ease of use and reduced operative time [9]. First-generation devices for all-inside meniscus repair with rigid fixators such as meniscal arrows, screw, and staples were associated with risks of implant-induced chondral damage and synovitis. Second-generation suture- and anchor-based devices were designed to minimize this risk as they leave only suture on the meniscal surface. There was also concern of inadequate fixation strength with first-generation meniscal fixators, especially for tears near the meniscocapsular junction. For this reason, Ahn had described modified all-inside suturing techniques using either posterolateral or posteromedial portals for tears near the meniscocapsular junction [2, 3]. However, recent studies have also demonstrated that second-generation sutureand anchor-based devices provide fixation comparable to the classic vertical mattress suture repair technique in terms of mean failure load and displacement after cyclic loading $[5,6]$. The FAST-FIX 360 device from Smith \& Nephew, used in this study, is one such device. Even when the all-inside repair is performed not vertically but horizontally (which is technically easier), the load to failure is not reduced [6]. However, in the case of an unstable and displaced BHMT, all-inside repair with either first- or second-generation devices cannot achieve satisfactory reduction or maintain that reduction. This proposed technique uses the inside-out reduction suture to achieve anatomic reduction and coaptation of the tear edges, yet avoids the disadvantages of conventional inside-out repairs. Stable fixation is then attained through all-inside meniscal repair with a secondgeneration suture-based device, before removal of the reduction suture. Gapping and puckering of the meniscus can be avoided by alternating the devices on both the femoral and tibial sides of the meniscus. All-inside meniscal repairs can be done in either a vertical or horizontal orientation.

This study has several limitations. Clinical outcomes for the proposed technique have not been studied, due to the relative infrequency of BHMT. Future prospective studies are warranted to study failure rates, functional outcomes, and complication rates with this technique. Also, recent systematic review comparing all-inside and inside-out meniscal repairs showed overall low levels of evidence and limited instruments for outcome measurement [8]. If subsequent high level evidence can show equivalent outcomes for all-inside and inside-out techniques, then cost effectiveness may favor inside-out repairs over all-inside repairs using meniscal fixators which tend to be expensive. However, the authors believe that most BHMT are best suited to all-inside repair as they usually extend to the posterior horn where inside-out repairs pose the risk of damage to the popliteal neurovascular bundle.

The proposed technique uses an inside-out suture to achieve and maintain anatomic reduction, followed by repair of a BHMT with second-generation all-inside meniscal fixators. To the best of the authors' knowledge, the combined use of an inside-out suture for meniscus 
reduction with all-inside repair of a BHMT is not a previously described technique. It potentially avoids the risks and morbidity of conventional inside-out repair, reduces operative time, and is easy to use.

\section{Conclusion}

The technique described is superior to existing techniques for the following reasons: (1) Reduction of the displaced meniscal tear is "extra-meniscal," avoiding further trauma to a damaged meniscus. (2) Tensioning of the two suture limbs created promotes better control of reduction through tensioning. (3) Risk of discomfort, infection, and neurovascular damage caused by a retained suture is reduced. (4) No additional portals/ equipment is required. We encourage this novel technique to be attempted by surgeons.

\section{Abbreviations}

ACL: Anterior cruciate ligament; BHMT: Bucket-handle meniscus tear; ROM: Range of motion

\section{Acknowledgements}

Nil

\section{Funding}

This study is institutionally funded.

\section{Availability of data and materials}

Please contact the authors for data requests.

\section{Authors' contributions}

All named authors in this manuscript played a critical role in the design and execution of the methodology and drafting of the manuscript. YJH and WW performed the novel surgical procedure. BK and YJH reviewed the scan images and drafted the manuscript. All authors read and approved the final manuscript.

\section{Authors' information}

WW is an Associate Professor and Head of Department at the National University Hospital (NUH), Singapore. BK and YJH are Orthopedic Trainees at the National University Hospital (NUH), Singapore. Please contact the authors' if further information is required.

\section{Ethics approval and consent to participate}

Domain Specific Review Board (DSRB) approval was sought and obtained.

\section{Consent for publication}

Consent to publish was obtained.

\section{Competing interests}

The authors declare that they have no competing interests.

\section{Publisher's Note}

Springer Nature remains neutral with regard to jurisdictional claims in published maps and institutional affiliations.

Received: 20 June 2017 Accepted: 15 November 2017

Published online: 04 December 2017

\section{References}

1. Ahn JH, Wang JH, Oh I. Modified inside-out technique for meniscal repair. Arthroscopy. 2004;20(Suppl 2):178-82.

2. Ahn JH, Kim SH, Yoo JC, Wang JH. All-inside suture technique using two posteromedial portals in a medial meniscus posterior horn tear. Arthroscopy. 2004;20(1):101-8.

3. Ahn JH, Oh I. Arthroscopic all-inside lateral meniscus suture using posterolateral portal. Arthroscopy. 2006;22(5):572.e1-4.
4. Albrecht-Olsen P, Kristensen G, Burgaard P, Joergensen U, Toerholm C. The arrow versus horizontal suture in arthroscopic meniscus repair: a prospective randomized study with arthroscopic evaluation. Knee Surg Sports Traumatol Arthrosc. 1999;7(5):268-73.

5. Barber FA, Herbert MA, Schroeder FA, Aziz-Jacobo J, Sutker MJ. Biomechanical testing of new meniscal repair techniques containing ultra high-molecular weight polyethylene suture. Arthroscopy. 2009;25(9):959-67.

6. Barber FA, Herbert MA, Bava ED, Drew OR. Biomechanical testing of suturebased meniscal repair devices containing ultrahigh-molecular-weight polyethylene suture: update 2011. Arthroscopy. 2012;28(6):827-34.

7. Fauno P, Nielsen AB. Arthroscopic partial meniscectomy: a long-term followup. Arthroscopy. 1992;8(3):345-9.

8. Fillingham YA, Riboh JC, Erickson BJ, Bach BR Jr, Yanke AB. Inside-out versus all-inside repair of isolated meniscal tears: an updated systematic review. Am J Sports Med. 2016:45(1):234-42.

9. Grant JA, Wilde J, Miller BS, Bedi A. Comparison of inside-out and all-inside techniques for the repair of isolated meniscal tears: a systematic review. Am J Sports Med. 2012;40(2):459-68.

10. Kocabey $\mathrm{Y}$, Taser $\mathrm{O}$, Nyland J, et al. Pullout strength of meniscal repair after cyclic loading: comparison of vertical, horizontal, and oblique suture techniques. Knee Surg Sports Traumatol Arthrosc. 2006;14(10):998-1003.

11. Miller MD, Thompson SR. Miller's review of orthopaedics. 7th ed. Philadelphia: Elsevier; 2016. p. 341-2.

12. Morgan CD. The "all-inside" meniscus repair. Arthroscopy. 1991:7(1):120-5.

13. Salata MJ, Gibbs AE, Sekiya JK. A systematic review of clinical outcomes in patients undergoing meniscectomy. Am J Sports Med. 2010;38(9):1907-16.
Submit your next manuscript to BioMed Central and we will help you at every step:

- We accept pre-submission inquiries

- Our selector tool helps you to find the most relevant journal

- We provide round the clock customer support

- Convenient online submission

- Thorough peer review

- Inclusion in PubMed and all major indexing services

- Maximum visibility for your research

Submit your manuscript at www.biomedcentral.com/submit
) Biomed Central 